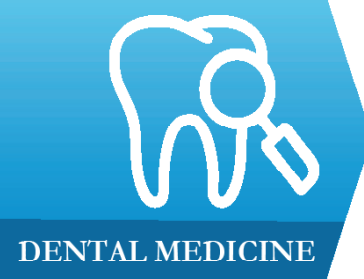

DENTAL MEDICINE

Department of Prosthodontics, Crown and Bridge, Hitkarini Dental College and Hospital, Jabalpur, Madhya Pradesh, India

DOI: $10.15386 / \mathrm{mpr}-1309$

Manuscript received: 18.02 .2019

Received in revised form: 15.05.2019

Accepted: 31.05.2019

Address for correspondence:

sukhadawagh.2@gmail.com

This work is licensed under a Creative Commons Attribution-NonCommercialNoDerivatives 4.0 International License

\section{Evaluation of maxillary anterior teeth proportion with Chu's Gauge in a population of Central India: an in vivo study}

Sukhada Arun Wagh, Sneha S. Mantri, Abhilasha Bhasin

\begin{abstract}
Aim. To evaluate the width/height proportions in maxillary anterior natural dentition and its correlation with Chu's esthetic proportion Gauge.

Settings and Design. Observational cross-sectional study with the inclusion criteria of well aligned maxillary anterior teeth.

Purpose. The present study attempts to perceive the application of Chu's Esthetic Proportion Gauge in a group of the central Indian population.

Methods. A total number of 150 participants within the age group 18-30 years were screened, out of which 80 participants were selected, who fulfilled the inclusion criteria of well aligned maxillary anterior teeth with pleasing smile, unrestored, periodontally sound, no fracture, no missing, absence of diastema teeth and no $\mathrm{H} / \mathrm{O}$ orthodontic treatment. The selected participants were seated on the dental chair with the Frankforts horizontal plane parallel to the floor. The participants were observed for height and width proportions using the Chu's esthetic proportion gauge and measured using digital vernier calliper. The proportion and measured height and width ratios were then compared.
\end{abstract}

Results. The recorded data was compiled and entered in a spreadsheet computer program (Microsoft Excel 2007) and then exported to data editor page of SPSS version 20.0 (SPSS Inc., Chicago, Illinois, USA). Intergroup comparison between gauge and vernier caliper was done using Chi-square test for proportions percentages (Qualitative data) and Mann-Whitney U test of width and height and then categorized into esthetic and unesthetic anterior teeth. Level of significance was set at 0.05 .

Conclusions. Within the limitations of the study, it was concluded that maxillary anterior teeth did show similarity with Chu's esthetic proportion scale.

Keywords: recurring esthetic dental proportion, proportion gauges, height/ width ratio, proportion scale

\section{Introduction}

Smile designing is a delicate blend of geometric principles and artistic abilities [1]. The increasing patient cosmetic demands have led to the development of multiple tools and technologies related to achieving esthetics in dental restoration [2]. Tooth size is one of the primary building blocks in the smile frame. As maxillary anterior are dominant teeth in the smile arch, the selection of correct tooth size allows arrangement of teeth in maxilla to enhance esthetics as well as treatment outcome [3].

Dental esthetics is influenced by different micro and macro esthetic factors which are inseparable and have impact on each other. Macro-esthetic features include tooth size, shape, form and proportion while tooth shade, color, texture, translucency are categorized under micro esthetics [4]. The restorative dentist must consider the patient's subjective concerns when designing a natural smile along with the objective criterion. 
Various tooth proportions are described in literature for the size of maxillary anterior teeth. The golden proportion is based on the theory that a relationship exists between two esthetically proportional parts. Fibonacci da Pisa, in the twelfth century, proposed the divine proportions or the Golden ratio [4]. This ratio was noted to be 1.618:1. Application of Golden proportion to dentistry was first introduced by Lombardini in 1973. It states that the width of maxillary lateral incisor, when viewed from front, should be in Golden proportion to the width of maxillary central incisor i.e $1: 1.618$ or $62 \%$. Thus, the width of maxillary lateral incisor to maxillary central incisor and the width of maxillary canine to lateral incisor should be $62 \%$ [5-8].

Methots proportion ("M"), is a modified Golden proportion. In Methots proportion the inter-molar distance of each patient, represents the width of the arch, and the width of the central insicors is used to determine the correct balance for the teeth displayed within that arch to create a pleasing smile. It can be seen that the modified ratio is 1.367 , as opposed to the Golden Rule of 1.618 [9].

Recurring esthetic dental (RED) proportion describes a constant ratio between the widths and height of maxillary anterior teeth. RED states that the widths of successive teeth when viewed from frontal aspect should remain constant as we move distally [10]. RED gives greater flexibility as it gives a range of proportion $62 \%$ to $80 \%$ [10]. Dr. Ward proposed the RED proportion for different heights of the maxillary anterior teeth $[11,12]$. Dr. Chu devised the tool based on the demographic study on the Caucasian population. The Chu's esthetic gauge is formed on the concept of Recurring Esthetic Dental proportion (RED) using the $78 \%$ RED proportion. It was designed to evaluate tooth size and proportion visually and objectively chair side [10-13].

Shetty et al. evaluated the existence of the RED proportion in natural dentition with pleasing smiles. They concluded that the RED proportion was not seen in the natural dentition of subjects with a pleasing smile [4].

Mootha et al. compared different recent tools such as DSD softwares, Chu's proportion Gauge, to evaluate the tooth proportions and concluded that the subjects with a pleasing smile were within the range of Chu's proportions scale and DSD [2].

Ozdemier et al. conducted a study to investigate the various proportions in the Turkish population and concluded that neither golden proportion, nor RED exist in natural dentition [14].

Kaisly et al. evaluated the golden proportion and width/height proportions in maxillary anterior dentition in Arabian and Kurdish population and detected no ideal ratio of width / height [15].

The variations in the reported studies may be result of ethnic characteristics specific to population studied.

Studies based on photogrammetric evaluation of RED and Golden proportion amongst the central Indian population were conducted by Deogade et al., Murthy et al., Meshram et al. and Ahmed et al [16-19]. The study concluded that no ideal ratio of width / height exist. Eversince the designing and clinical use of the gauge there scarcity of literature proving its validity.

Maxillary anterior teeth being the dominant teeth in the smile frame, the chair side evaluation of proportion using Chu's proportion gauge in the central Indian population as a measure of esthetic was the focus of the present study.

The purpose of the study was to evaluate whether the average dimensions obtained for width and height of maxillary anterior teeth are similar to the values proposed by Chu's gauge, which is based on $78 \%$ RED, so that the gauge can be used for rehabilitation of maxillary anterior teeth for the focus demographic population.

The study aimed to evaluate subsistence of Chu's esthetic proportion scale and width / length ratio in pleasing smiles in central India with the objectives:

- To evaluate and compare the dimensions of maxillary central (CI) bilaterally with Chu's proportion scale and digital vernier.

- To evaluate and compare the dimensions of lateral central (LI) bilaterally with Chu's proportion scale and digital vernier.

- To evaluate and compare the dimensions of canine $(\mathrm{Cn})$ bilaterally with Chu's proportion scale and digital vernier.

The null hypothesis is that the natural maxillary anterior teeth proportions did not follow the Chu's proportion scale.

\section{Materials and methods}

The study was performed in Department of Prosthodontics and Crown \& Bridges. The study was approved by the institutional ethical committee and informed consent was obtained from the participants. A total of 150 participants within the age group 18-30 years were evaluated, out of which 80 were selected who met the inclusion criteria of pleasing smiles with well aligned maxillary anterior teeth, unrestored, periodontally sound, no fracture, no missing, absence of diastema in maxillary anterior teeth and no $\mathrm{H} / \mathrm{O}$ orthodontic treatment following the convenience sampling.

\section{Procedure}

The height and width of maxillary anteriors were measured intraorally using two methods:

1. Firstly, using the ' $T$ ' bar tip of the Chu's esthetic proportion gauges.

2. Secondly, using the digital vernier caliper.

Method of evaluation using the Chu's esthetic proportion gauge

Chu's esthetic proportion gauge is a set of one handle and four colour coded tips, the $\mathrm{T}$ bar tip, the inline tip, the papilla gauge and the bone sounding gauge (Figure 1). 


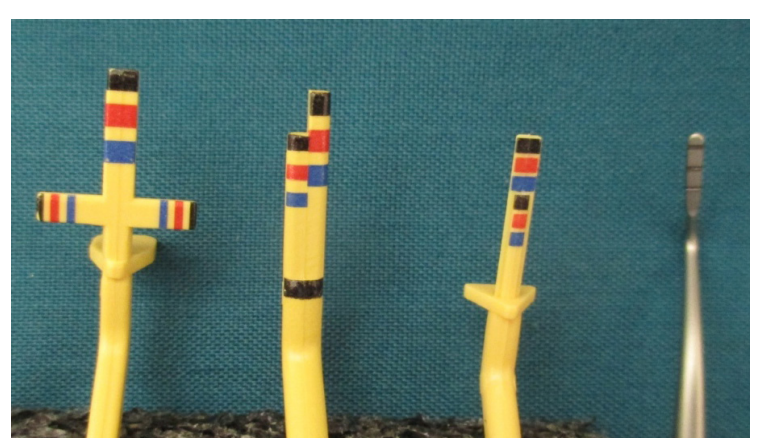

Figure 1. Set of Chu's Esthetic Proportion Gauges.

In this study, $\mathrm{T}$ bar tip was selected, taking into consideration the inclusion criteria i.e. well aligned maxillary anterior teeth. The other tips of the set namely: Inline tip, papilla gauge and bone sounding gauge are used for measurements of crowded dentition, interdental papilla and supracrestal tissue respectively. T bar tip is ' $\mathrm{T}$ ' shaped and has color coded bands with preset height/ width ratio (Table I and Figure 2) viz red, blue and yellow on its vertical bar (height measurements) and the horizontal bar (width measurements) which correspond to each other [2]. Height and width dimensions are measured simultaneously. The bands are $1.5 \mathrm{~mm}$ thick, which covers the $78 \%$ of Recurring Eesthetic Dental proportion (RED). These bands are at a distance of $1 \mathrm{~mm}$ from each other. For example, the central incisor, with "red" width 8.5 $\mathrm{mm}$ will be in proper proportion if its height is also "red" height $11 \mathrm{~mm}$ (Figure 2).

All the readings were noted with each participant seated, head-supported and with Frankfort's horizontal plane parallel to the ground, on the dental chair. Cheek retractor was used for better visualization and accessibility. An approximate midpoint of each anterior tooth was marked with a colored marker on the incisal edges. The gauge had an incisal stop, which stabilized and helped to orient the gauge onto the tooth surface. This stop was approximated at the marked midpoint along the long axis of the tooth. The tooth was first evaluated for width using color coded markings (Central incisor $=$ Red color, Lateral incisor $=$ Blue color and Canine $=$ Intermediate yellow Color) $7 \mathrm{~mm}$ away from the incisal edge. Simultaneously, the height from the incisal edge to the zenith point on gingival margin was assessed using the corresponding color codings. One tooth at a time was evaluated with Chu's esthetic gauge for height and width proportions (Figure 3). According to the gauge, the tooth is supposed to be in proportion if the color codings on the vertical and horizontal bar match each other. The obtained proportions were then tabulated.

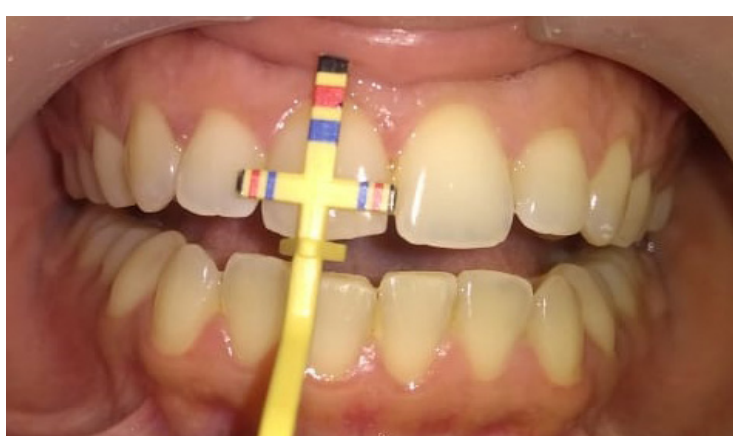

Figure 3. Participant evaluated with T bar tip.

Table I. Average range values for height and width on Chu's T bar gauge.

\begin{tabular}{|c|c|}
\hline Colour codes on the instrument & Significance (average range of values of maxillary teeth lengths and widths in $\mathrm{mm}$ ) \\
\hline Red & Incisor (avg $L=9.5$ to 11$)$ and (avg $W=7.5$ to 8.5 ) \\
\hline Blue & Lateral incisor $(\operatorname{avg} \mathrm{L}=7$ to 8.5$)(\operatorname{avg} \mathrm{W}=5.5$ to 6.5$)$ \\
\hline Yellow & Canine $(\operatorname{avg} L=8.5$ to 9.5$)(\operatorname{avg~} \mathrm{W}=8.5$ to 9.5$)$ \\
\hline
\end{tabular}
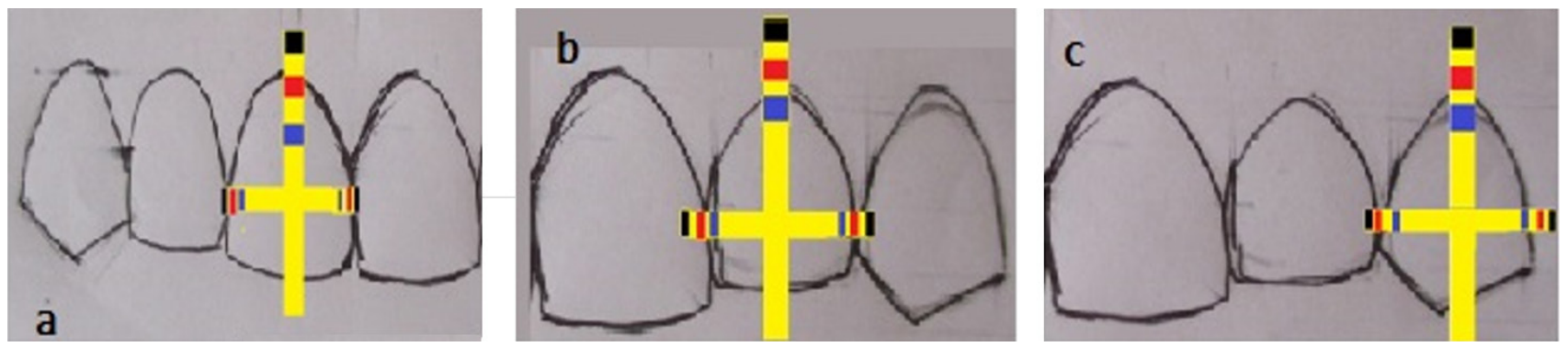

Figure 2. T bar tip with colour coded bands for a) cental incisor, b) lateral incisor, c) canine. 
Similarly, Digital vernier calliper, calibrated in millimeters (mm) up to $0.01 \mathrm{~mm}$ accuracy, was used to record the exact height and width dimensions of maxillary anterior teeth at the approximately same guidances that of proportion gauge i.e, width was measured $7 \mathrm{~mm}$ apical to the incisal edge and height was measured from marked midpoint to the zenith point. All the measurements were done by a single examiner to eliminate interobserver error. All the dimensions were measured three times for each tooth and the average was recorded in order to minimize the error. The observed readings were tabulated. The proportions deduced from the gauge as well as from the digital venier calliper measurements were compared.

\section{Statistical analysis}

The recorded data weres compiled and entered in a spreadsheet computer program (Microsoft Excel 2007) and then exported to data editor page of SPSS version
20.0 (SPSS Inc., Chicago, Illinois, USA). Intergroup comparison between gauge and vernier caliper was done using Chi- square test for proportions and percentages (Qualitative data) of width and height and then categorized into proportionate and disproportionate anterior teeth. Level of significance was set at 0.05 .

\section{Results}

Table II shows the comparison of height/ width proportion of different teeth. Width/height proportions of central incisor, lateral incisors and canine were significantly higher $(\mathrm{P}<0.001)$.

According to the statistical analysis, the proportion obtained from the vernier caliper measurements were $83.272 \pm 11.01$ for central incisors, $82.856 \pm 12.26$ for lateral incisors and $79.613 \pm 12.25$ for canines, which did not corelate with Chu's proportion scale $(78.00 \pm 0.5)$.

Table II. Comparison of gauge and vernier caliper.

\begin{tabular}{|c|c|c|c|c|}
\hline \multicolumn{5}{|c|}{ Right central incisor } \\
\hline Measurement technique & $\begin{array}{c}\text { Esthetic } \\
\text { N(\%) }\end{array}$ & $\begin{array}{c}\text { Unesthetic } \\
\text { N(\%) }\end{array}$ & $\begin{array}{l}\text { Total } \\
\text { N(\%) }\end{array}$ & p-value \\
\hline Chu's Gauge & $50(62.5)$ & $30(37.5)$ & $80(100)$ & \multirow[b]{2}{*}{$0.001^{* *}$} \\
\hline Vernier Caliper & $13(16.25)$ & $67(83.75)$ & $80(100)$ & \\
\hline \multicolumn{5}{|c|}{ Left central incisor } \\
\hline Measurement technique & $\begin{array}{c}\text { Esthetic } \\
\text { N(\%) }\end{array}$ & $\begin{array}{c}\text { Unesthetic } \\
\mathbf{N}(\%)\end{array}$ & $\begin{array}{l}\text { Total } \\
\text { N(\%) }\end{array}$ & p-value \\
\hline Chu's Gauge & $53(66.25)$ & $27(33.75)$ & $80(100)$ & \multirow{2}{*}{$0.001^{* *}$} \\
\hline Vernier Caliper & $13(16.25)$ & $67(83.75)$ & $80(100)$ & \\
\hline \multicolumn{5}{|c|}{ Right lateral incisor } \\
\hline Measurement technique & $\begin{array}{c}\text { Esthetic } \\
\mathbf{N}(\%)\end{array}$ & $\begin{array}{c}\text { Unesthetic } \\
\text { N(\%) }\end{array}$ & $\begin{array}{l}\text { Total } \\
\text { N(\%) }\end{array}$ & p-value \\
\hline Chu's Gauge & $45(56.25)$ & $35(43.75)$ & $80(100)$ & \multirow{2}{*}{$0.001^{* *}$} \\
\hline Vernier Caliper & $12(15)$ & $68(85)$ & $80(100)$ & \\
\hline \multicolumn{5}{|c|}{ Left lateral incisor } \\
\hline Measurement technique & $\begin{array}{c}\text { Esthetic } \\
\text { N(\%) }\end{array}$ & $\begin{array}{c}\text { Unesthetic } \\
\mathrm{N}(\%)\end{array}$ & $\begin{array}{l}\text { Total } \\
\text { N(\%) }\end{array}$ & p-value \\
\hline Chu's Gauge & $46(81.25)$ & $34(18.75)$ & $80(100)$ & \multirow{2}{*}{$0.001^{* *}$} \\
\hline Vernier Caliper & $12(15)$ & $68(85)$ & $80(100)$ & \\
\hline \multicolumn{5}{|c|}{ Right canine } \\
\hline Measurement technique & $\begin{array}{c}\text { Esthetic } \\
\text { N(\%) }\end{array}$ & $\begin{array}{c}\text { Unesthetic } \\
\mathbf{N}(\%)\end{array}$ & $\begin{array}{l}\text { Total } \\
\text { N(\%) }\end{array}$ & p-value \\
\hline Chu's Gauge & $46(81.25)$ & $34(18.75)$ & $80(100)$ & \multirow{2}{*}{$0.001^{* *}$} \\
\hline Vernier Caliper & $17(21.25)$ & $63(78.75)$ & $80(100)$ & \\
\hline \multicolumn{5}{|c|}{ Left canine } \\
\hline Measurement technique & $\begin{array}{c}\text { Esthetic } \\
\mathbf{N}(\%)\end{array}$ & $\begin{array}{c}\text { Unesthetic } \\
\text { N(\%) }\end{array}$ & $\begin{array}{l}\text { Total } \\
\text { N(\%) }\end{array}$ & p-value \\
\hline Chu's Gauge & $45(56.25)$ & $35(43.75)$ & $80(100)$ & \multirow{2}{*}{$0.001^{* *}$} \\
\hline Vernier Caliper & $17(21.25)$ & $63(78.75)$ & $80(100)$ & \\
\hline
\end{tabular}

Test applied: Chi square test; ${ }^{* *}$ p-value $=0.001$ (highly significant).

Table III. Comparison of height / width proportions between right and left side teeth.

\begin{tabular}{l|c|c|c}
\multirow{2}{*}{ Tooth } & \multicolumn{2}{|c|}{ Side } & Mann-Whitney U test \\
\cline { 2 - 4 } & Right & Left & MW=1243.500, $\mathrm{P}=0.590(>0.05)$, Not sig. \\
Central Incisor & $83.61 \pm 12.06$ & $82.40 \pm 12.05$ & $\mathrm{MW}=1023.500, \mathrm{P}=0.927(>0.05)$, Not sig. \\
Lateral Incisor & $82.32 \pm 15.41$ & $80.88 \pm 15.22$ & $\mathrm{MW}=977.500, \mathrm{P}=0.647(>0.05)$, Not sig. \\
Canine & $79.17 \pm 17.20$ & $79.51 \pm 15.03$ &
\end{tabular}


Table III shows comparisons of standard gauge proportion values of teeth on right and left side teeth in the study population. On comparison there was no significant difference for right and left side values of central incisor, lateral incisor and canine. The results of the investigation showed that the height and width values for left and right side did not show significant difference in their measurements where $P$ value was found to be $P<$ 0.05 (Table III). It could be attributed that they were equal or approximately equal in height and width to each other.

\section{Discussion}

"No human inquiry can be called science unless it pursues its path through mathematical exposition and demonstration" Leonardo da Vinci [6].

The distinction between pleasing and non pleasing smile is a subjective process. Therefore dental professionals dealing with patients' esthetic demands need to rely on fulfilling esthetic principles proved valid for clinical application.

Various methods were used to clinically evaluate the existence of the proposed esthetic proportions. These methods included static method, photogrammetric method and digital method for evaluation. In the present study direct intraoral measurement was done using Chu's proportion Gauge and Digital vernier caliper.

A study conducted by Ward evaluating the existence of the RED proportion in small group of North American population using Digital computer software, showed no existence of RED proportion in natural pleasant smile $[10,11]$. The results were similar to the study conducted by Sandeep N et al. who concluded that RED proportion was not seen in natural dentition and width of maxillary anterior teeth followed the golden percentage by Snow [20].

The results of the present study supported earlier literatures, concluding that RED proportion did not exist in the sample population. In the present study the vernier caliper measurements were $83.272 \pm 11.01$ for central incisors, $82.856 \pm 12.26$ for lateral incisors and $79.613 \pm 12.25$ for canines, which were not in accordance with the Chu's proportion Gauge (78.00土0.5).

The results of the study are in agreement with the study conducted by Shetty et al, Murthy et al, and Ahmed $\mathrm{N}$ et al. which stated that there was no relevance of mathematical proportion i.e. RED proportion in natural dentition. The anterior teeth proportions of the study population did not show co-relation with RED proportion [4-18].

The study conducted by Mootha et al. comparing DSD protocol, which is based on the RED concept and Gauge Proportions for maxillary anterior teeth, confirmed that they are co-related and RED proportion existed [1]. The results were in contradiction to the present study .
This study was one of the first of its kind using direct static intra-oral measurement of teeth size for both width and height parameters eliminating errors of photogrammetric method.

The limitations of the study were that it was conducted on a small sample size and also gender wise distribution was not considered. Restricted population is a limitation which questions the applications of results to a wider range of population. Future studies evaluating gender, ethnicity, mandibular teeth, posterior teeth in larger sample size is recommended. The null hypothesis was rejected.

\section{Conclusion}

Within the limitations of the study the following conclusion were drawn.

The average dimensions of natural maxillary anterior teeth in pleasing smile for width/height proportion did not simulate $78 \%$ RED proportion applied by Chu's gauge in the studied population.

On evaluation and comparison with Chu's proportion scale and digital vernier caliper the anterior teeth were not found in esthetic proportion. Hence Chu's esthetic proportion scale did not show any similarity to the natural teeth dimensions in the Central Indian population .

\section{Clinical implication}

Although the Chu's esthetic scale did not show relevance in the natural dentition of studied pooulation, it can be one of the chair side modality in esthetic dental procedures.

\section{References}

1. Galip Gruel Smile designing. In The science and art of porcelain laminates and veneers. Quintessence Publishing Co. Ltd. 2003; pp. 58-109.

2. Mootha A, Jaiswal S. Evaluation of maxillary anterior teeth and their relation to the various geometric proportions in Indian population sample. J Interdiscip Dentistry. 2018;8:6267.

3. Chu SJ. Range and mean distribution frequency of individual tooth width of the maxillary anterior dentition. Pract Proced Aesthet Dent. 2007;19:209-215.

4. Shetty S, Pitti V, Satish Babu C, Surendra Kumar G, Jnanadev K. To evaluate the validity of Recurring Esthetic Dental propotion in natural dentition. J Conserv Dent. 2011;14:314-317.

5. Laxmikanth SM, Raghavendra SR. Golden proportion: A review. J Adv Clin Res Insights. 2014;1:25-29.

6. Levin EI. Dental esthetics and the golden proportion. J Prosthet Dent. 1978;40:244-252.

7. Preston JD. The golden proportion revisited. J Esthet Dent. 1993;5:247-251.

8. Lombardi RE. The principles of visual perception and their 
clinical applications to denture esthetics. J Prosthet Dent. 1973;29:358-382.

9. Methot A. M proportion: A new golden rule in dentistry. Canad J Cosmet Dent. 2010:34-40.

10. Ward DH. A study of dentists' preferred maxillary anterior tooth width proportions: comparing the recurring esthetic dental proportion to other mathematical and naturally occurring proportions. J Esthet Restor Dent. 2007;19:324337.

11. Ward DH. Proportional smile design: using the recurring esthetic dental proportion to correlate the widths and lengths of the maxillary anterior teeth with the size of the face. Dent Clin North Am. 2015;59:623-638.

12. Chu SJ. Biometric approach to aesthetic crown lengthening. Prac Proced Aesthet Dent. 2008;402-408.

13. Stephen $\mathrm{C}$, Fletcher P, Mieleszko A. Clinical application of innovative measurement gauges for predictable correction of tooth size/proportion and gingival architecture discrepancies. Quintessence Dent Tech. 2009;63-76.

14. Özdemir H, Köseoğlu M, Bayindir F. An investigation of the esthetic indicators of maxillary anterior teeth in young Turkish people. J Prosthet Dent. 2018;120:583-588.
15. Al-Kaisy N, Garib BT. Analysis of the golden proportion and width/height ratios of maxillary anterior teeth in Arab and Kurdish proportions. J Prosthet Dent. 2018;119:981-986.

16. Deogade SC, Mantri SS, Sumathi K, Rajoriya S. The relationship between innercanthal dimension and interalar width to the intercanine width of maxillary anterior teeth in central Indian population. J Indian Prosthodont Soc. 2015;15:91-97.

17. Murthy BV, Ramani N. Evaluation of natural smile: Golden proportion, RED or Golden percentage. J Conserv Dent. 2008;11:16-21.

18. Meshramkar R, Patankar A, Lekha K, Nadiger R. A study to evaluate the prevalance of golden proportion and RED proportion in aesthetically pleasing smiles. Eur J Prosthodont Restor Dent. 2013;21:29-33.

19. Ahmed N, Abbas M, Maqsood A. Evaluation of recurring esthetic dental proportion in natural smile of Pakistani sample. Pakistan Oral \& Dental Journal. 2014;34:739-742.

20. Sandeep N, Satwalekar P, Srinivas S, Reddy CS, Reddy GR, Reddy BA. An Analysis of Maxillary Anterior Teeth Dimensions for the Existence of Golden Proportion: Clinical Study. J Int Oral Health. 2015;7:18-21. 